\title{
Adaptive Fault-Tolerant Cruise Control for a Class of High-Speed Trains with Unknown Actuator Failure and Control Input Saturation
}

\author{
Tao Tao and Hongze Xu \\ School of Electronic and Information Engineering, Beijing Jiaotong University, Beijing 100044, China \\ Correspondence should be addressed to Tao Tao; tttt23456@live.cn
}

Received 18 February 2014; Revised 9 June 2014; Accepted 15 June 2014; Published 8 July 2014

Academic Editor: Cristian Toma

Copyright (C) 2014 T. Tao and H. Xu. This is an open access article distributed under the Creative Commons Attribution License, which permits unrestricted use, distribution, and reproduction in any medium, provided the original work is properly cited.

\begin{abstract}
This paper investigates the position and velocity tracking control of a class of high-speed trains (HST) with unknown actuator failures (AF) and control input saturation (CIS). Firstly, a nonlinear dynamic model for HST at normal operating status is built. The structure of traction system in HST is analyzed and the corresponding model for HST with unknown AF is presented as well. The type of AF under consideration is that some of the plant inputs are influenced by hopping function. An adaptive model-based fault detection and diagnosis (AMFDD) module is proposed based on immersion and invariance (I\&I) method to make decisions on whether a fault has occurred. A new framework to design a monotone mapping is proposed in I\&I method, that is, $P(x)$-monotone. Using on-line obtained fault information, an adaptive law is designed to update the controller parameters to handle unknown AF and CIS in HST simultaneously when some of plant parameters are unknown. Closed-loop stability and asymptotic position and velocity tracking are ensured. Numerical simulations of China Railways High-speed 2 (CRH2) train are provided to verify the effectiveness of the presented scheme.
\end{abstract}

\section{Introduction}

In practical applications, nonlinearity caused by imperfections of modelling leads to performance degradation of the closed-loop system. Compensation control of nonlinear systems has been a research topic of wide interest. In [1], unknown continuous and monotone nonlinearities which satisfy some constraints are considered, and an adaptive controller using artificial neural networks is proposed for a class of nonlinear systems with input nonlinearities. In [2], direct adaptive neural network control is presented for uncertain multi-input/multi-output nonlinear systems in block triangular forms. Reference [3] presents two indirect adaptive control schemes which are employed to approximate unknown nonlinear functions. Reference [4] proposes a robust control algorithm for a three-axis stabilized flexible spacecraft in the presence of control input nonlinearity/deadzone. In $[5,6]$, an adaptive neurofuzzy control is proposed by describing nonlinear affine systems as a Takagi-Sugeno fuzzy model. An adaptive fuzzy backstepping control approach is considered in [7] for a class of nonlinear strict-feedback systems with unknown functions, unknown dead zones, and immeasurable states. In [8], step tracking control problem for discrete-time nonlinear systems, which are represented by a Takagi-Sugeno fuzzy system, is investigated in a networked environment with a limited capacity. In [9], the problem of adaptive fuzzy tracking control via output feedback for a class of uncertain strict-feedback nonlinear systems with unknown time-delay functions is investigated. In [10], the problem of tracking control for a class of large-scale nonlinear systems with unmodeled dynamics is addressed by designing the decentralized adaptive fuzzy output feedback controller to guarantee that all the signals in the closed-loop system are bounded. Reference [11] deals with the adaptive sliding-mode control problem for nonlinear active suspension systems with varying sprung and unsprung masses, unknown actuator nonlinearity, and suspension performances.

In HST, besides nonlinearity, train traction module may fail to work due to various kinds of reasons [12-14], such as overvoltage in traction transformer, overcurrent in traction 
converter, and overheat in asynchronous motor. AF in HST will cause problems, that is, inaccurate position tracking, inaccurate velocity tracking, or even train accident. It is significant to design the controller addressing AF to realize fault-tolerant control (FTC) in HST [15-21]. In control society, there are many remarkable results on FTC for AF. In [22], a class of nonlinear systems with faults, parametric uncertainties, and without full state measurements are considered. A novel observer is designed whose estimation error is not affected by faults and an observer-based faulttolerant tracking controller is proposed to make the outputs asymptotically track the reference signals while the states are bounded. In [23], a FTC scheme based on the adaptive control technique for near-space-vehicle attitude dynamics is considered. In [24], a FTC scheme using backstepping and neural network methodology is proposed for a class of nonlinear systems with known structure and unknown faults. In $[25,26]$, a direct adaptive feedback controller is developed for linear time-invariant plants with AF and the closed-loop stability and asymptotic tracking are ensured. Reference [27] proposes an adaptive compensation for parametric strictfeedback systems. A fault-tolerant robust control for a class of nonlinear systems is investigated in [28]. A robust FTC will switch itself between robust control strategies designed under normal operation and faulty condition. Reference [29] investigates the reliable $H_{\infty}$ control problem for discrete-time piecewise linear systems with time delays and AF. In [30], a fault-tolerant controller is presented for Lipschitz nonlinear continuous-time systems in the presence of disturbances and noises. An integrated design of the adaptive robust control and the fault identification for a linear system with $\mathrm{AF}$ is proposed in [31]. Reference [32] investigates the FTC problem for near-space vehicle attitude dynamics with $\mathrm{AF}$, which is described by a Takagi-Sugeno fuzzy model. In [33], a new FTC scheme is proposed by incorporating integral sliding modes, unknown input observers, and a fixed control allocation scheme, where only measured system outputs are assumed to be available. The problem of FTC for a class of nonlinear systems with AF is discussed, and an observer-based FTC scheme is proposed in [34]. Adaptive fuzzy observers are proposed to provide a bank of residuals for fault detection and isolation and an accommodation scheme is proposed to compensate for the effect of the fault. Reference [35] presents a FTC for nonlinear systems which are connected in a networked control system.

CIS, which implies that the output of serving motors is constrained, is another problem in HST, and a few studies have been done on this field [36, 37]. Study on designing controllers by considering CIS beforehand is promoted for system in which higher performance is expected. Karason and Annaswamy [38] deal with the problem of adaptive control for a linear time-invariant plant in the presence of constraints on the input amplitude. In [39], a nonlinear small gain theorem is presented that provides formalism for analyzing the behavior of certain control systems that contain or utilize saturation. Polycarpou et al. [40] address the issue of CIS in on-line approximation based control for nonlinear systems, and a modified control design framework is presented for preventing CIS from destroying the learning capabilities and memory of an on-line approximation. In [41], the robust control of an induction motor is investigated, and a parameter-dependent model is addressed. Wu et al. [42] present a method, for designing output feedback laws that stabilize a linear system subject to actuator saturation with a large domain of attraction, which applies to general linear systems including strictly unstable ones and is presented in both continuous-time and discrete-time setting. Zhou et al. [43, 44] develop some significative methods in this direction using Raccati equations as a basic tool. Fridman and Dambrine [45] consider quantized and delayed state feedback control of linear systems with given constant bounds on the quantization error and on the time-varying delay. Reference [46] deals with the problem of tracking and stabilization control of internally damped mobile robots with unknown parameters and subject to input torque saturation and external disturbances.

Recently, a novel I\&I adaptive method for nonlinear systems is presented to realize performance-oriented control [47-49]. In this paper, based on a nonlinear dynamic model for a class of HST, an adaptive FTC with I\&I AMFDD is presented. An AMFDD module using I\&I adaptive state observer is designed to detect AF, and direct adaptive controller based on on-line AMFDD information is achieved to handle nonlinearity, unknown AF, and CIS in HST. A new framework to design a monotone mapping is proposed in I\&I method, that is, $P(x)$-monotone. The stability of HST systems is proved theoretically.

The rest of this paper is organized as follows. Section 2 introduces problem formulation in HST. In Section 3, an AMFDD module is introduced and corresponding I\&I adaptive FTC is presented for AF and CIS. The simulation is shown in Section 4. Section 5 draws the conclusion.

\section{Problem Formulation}

In this section, we propose a nonlinear dynamic model for a class of HST with and without AF.

2.1. Dynamic Model of a Single Carriage. The HST consists of a number of carriages, and couplers are used to couple adjacent carriages. A carriage during travelling is subjected to various kinds of forces, such as traction and braking forces, forces between carriages, and resistance forces. The force analysis of a single carriage in HST is illustrated in Figure 1.

Traction and Braking Forces. $u_{i}$ denotes traction force of the $i$ th carriage, that is, $u_{i}>0$, and braking force of the $i$ th carriage, that is, $u_{i}<0$. $\lambda_{i}$ denotes whether the $i$ th carriage is powered or nonpowered.

Assumption 1. The class of HST that we considered is distributed driving type [50], that is, $\lambda_{i}=1$ for all carriages.

Interacting Forces between Carriages. Connection modules between two carriages in HST are nonlinear subsystems. Hence, it is difficult to obtain accurate mechanical model of interacting forces between two carriages. A widely used 




FIgURE 1: The force analysis of a single carriage.

mechanical model for couplers consists of elastic forces and damping forces. In [51], nonlinear elastic force acting between carriages is described as follows:

$$
f_{k}(t)=k(t) \epsilon_{i}(t),
$$

where relative displacement between two carriages is as

$$
\epsilon_{i}(t)=x_{i}(t)-x_{i-1}(t),
$$

where $x_{i}(t)$ is position of the $i$ th carriage. $k(t)$ denotes nonlinear relationship between displacements and forces and

$$
k(t)=k_{0}\left(1+\epsilon_{i}(t) \sigma\right) .
$$

If $\sigma=0$, coupler between carriages is linear, that is, $k(t)=k_{0}$, which does not exist in practice. If $\sigma<0$ or $\sigma>0$, the coupler is soft coupling or hard coupling, respectively. Khalil and Grizzle [52] and Franklin and Powell [53] have proved that a system with a soft spring is more likely to have unsatisfying control performance. We take the minimum value $k$ of $k(t)$ in controller design, that is, $k(t)=k$. The damping force is described as

$$
f_{b}(t)=b(t)\left(\dot{x}_{i}(t)-\dot{x}_{i-1}(t)\right),
$$

where $b(t)$ is damping coefficient and $\dot{x}_{i}(t)$ is velocity of the $i$ th carriage. We take the minimal value $b$ of the coefficient $b(t)$, that is, $b(t)=b$. The interacting force between carriages is as

$$
f_{i}^{c}(t)=f_{k}(t)+f_{b}(t)
$$

Resistance Forces of HST. During travelling, carriages of HST are subjected to various kinds of resistance forces mainly including mechanical resistance and air resistance. The two forces are as follows [54]:

$$
f_{i}^{p}(t)=m_{i}\left(c_{0}+c_{v} \dot{x}_{i}(t)+c_{a} \dot{x}_{i}^{2}(t)\right),
$$

where $m_{i}$ represents mass of the $i$ th carriage and $m_{i}\left(c_{0}+\right.$ $\left.c_{v} \dot{x}_{i}(t)\right)$ and $m_{i} c_{a} \dot{x}_{i}^{2}(t)$ denote mechanical resistance and air resistance, respectively. $c_{0}, c_{v}$, and $c_{a}$ are coefficients which may not be obtained accurately during train locomotion.

Slope and curve resistance forces of HST depend on railway condition. Slope and curve resistance forces are normally considered by Garg and Dukkipati [55] as follows:

$$
f_{i}^{e}(t)=m_{i} g \sin \theta_{i}+0.002 \frac{m_{i} d_{a}}{R_{t}},
$$

where $d_{a}$ is axle length of a carriage and $R_{t}$ is turning radius. Based on the above analysis, dynamic model of a single carriage in HST is given as follows:

$$
\begin{aligned}
m_{i} \ddot{x}_{i}(t)= & \lambda_{i} u_{i}(t)+f_{i+1}^{c}(t)-f_{i}^{c}(t)-f_{i}^{p}(t)-f_{i}^{e}(t) \\
= & \lambda_{i} u_{i}(t)-m_{i}\left(c_{0}+c_{v} \dot{x}_{i}(t)\right)-m_{i} c_{a} \dot{x}_{i}^{2}(t) \\
& -k\left(x_{i}(t)-x_{i-1}(t)\right)-b\left(\dot{x}_{i}(t)-\dot{x}_{i-1}(t)\right) \\
& -k\left(x_{i}(t)-x_{i+1}(t)\right)-b\left(\dot{x}_{i}(t)-\dot{x}_{i+1}(t)\right) \\
& -m_{i} g \sin \theta_{i}-0.002 \frac{m_{i} d_{a}}{R_{t}} .
\end{aligned}
$$

2.2. Dynamic Model of HST. Dynamic model of HST with $n$ carriages can be derived as follows:

$$
\begin{aligned}
m_{1} \ddot{x}_{1}(t)= & \lambda_{1} u_{1}(t)-k\left(x_{1}(t)-x_{2}(t)\right) \\
& -b\left(\dot{x}_{1}(t)-\dot{x}_{2}(t)\right)-m_{1}\left(c_{0}+c_{v} \dot{x}_{1}(t)\right) \\
& -m_{1} c_{a} \dot{x}_{1}^{2}(t)-m_{1} g \sin \theta_{1}-0.002 \frac{m_{1} d_{a}}{R_{t}}, \\
m_{2} \ddot{x}_{2}(t)= & \lambda_{2} u_{2}(t)-k\left(x_{2}(t)-x_{1}(t)\right) \\
& -b\left(\dot{x}_{2}(t)-\dot{x}_{1}(t)\right)-k\left(x_{2}(t)-x_{3}(t)\right) \\
& -b\left(\dot{x}_{2}(t)-\dot{x}_{3}(t)\right)-m_{2}\left(c_{0}+c_{v} \dot{x}_{2}(t)\right) \\
& -m_{2} c_{a} \dot{x}_{2}^{2}(t)-m_{2} g \sin \theta_{2}-0.002 \frac{m_{2} d_{a}}{R_{t}}, \\
m_{i} \ddot{x}_{i}(t)= & \lambda_{i} u_{i}(t)-k\left(x_{i}(t)-x_{i-1}(t)\right) \\
& -b\left(\dot{x}_{i}(t)-\dot{x}_{i-1}(t)\right)-k\left(x_{i}(t)-x_{i+1}(t)\right) \\
& -b\left(\dot{x}_{i}(t)-\dot{x}_{i+1}(t)\right)-m_{i}\left(c_{0}+c_{v} \dot{x}_{i}(t)\right) \\
& -m_{i} c_{a} \dot{x}_{i}^{2}(t)-m_{i} g \sin \theta_{i}-0.002 \frac{m_{i} d_{a}}{R_{t}}, \\
m_{n} \ddot{x}_{n}(t)= & \lambda_{n} u_{n}(t)-k\left(x_{n}(t)-x_{n-1}(t)\right) \\
& -b\left(\dot{x}_{n}(t)-\dot{x}_{n-1}(t)\right)-m_{n}\left(c_{0}+c_{v} \dot{x}_{n}(t)\right) \\
& -m_{n} c_{a} \dot{x}_{n}^{2}(t)-m_{n} g \sin \theta_{n}-0.002 \frac{m_{n} d_{a}}{R_{t}}, \\
& \vdots
\end{aligned}
$$


where $x_{i}$ subsystem represents dynamics of the $i$ th carriage in HST. To simplify the controller design, (9) is rewritten as

$$
\begin{array}{rl}
\dot{x}(t)= & A x(t)+B u(t)+C_{0}+C_{v} x(t)+C_{a} x^{2}(t) \\
= & {\left[\begin{array}{cc}
0_{n \times n} & A_{n \times n} \\
A_{21} & A_{22}
\end{array}\right] x(t)+\left[\begin{array}{c}
0_{n \times n} \\
B_{2}
\end{array}\right] u(t)+\left[\begin{array}{c}
0_{n \times 1} \\
E_{02}+C_{02}
\end{array}\right]} \\
& +\left[\begin{array}{ll}
0_{n \times n} & 0_{n \times n} \\
0_{n \times n} & C_{v 2}
\end{array}\right] x(t)+\left[\begin{array}{ll}
0_{n \times n} & 0_{n \times n} \\
0_{n \times n} & C_{a 2}
\end{array}\right] x^{2}(t), \\
y & C x(t),
\end{array}
$$

where $x=\left[\begin{array}{llllll}x_{1} & \cdots & x_{n} & \dot{x}_{1} & \cdots & \dot{x}_{n}\end{array}\right]^{\mathrm{T}}$ is the state vector. $A \in R^{2 n \times 2 n}$ and $B \in R^{2 n \times n}$ are known, and $C_{0} \in R^{2 n}, C_{v} \in$ $R^{2 n \times 2 n}$, and $C_{a} \in R^{2 n \times 2 n}$ are unknown matrixes, and we have

$$
\begin{aligned}
& A_{12}=\left[\begin{array}{ccccccc}
\frac{-k}{m_{1}} & \frac{k}{m_{1}} & 0 & \cdots & \cdots & \cdots & 0 \\
\frac{k}{m_{2}} & \frac{-2 k}{m_{2}} & \frac{k}{m_{2}} & 0 & \cdots & \cdots & 0 \\
0 & \frac{k}{m_{3}} & \frac{-2 k}{m_{3}} & \frac{k}{m_{3}} & 0 & \cdots & 0 \\
0 & \cdots & \cdots & \cdots & \cdots & \cdots & 0 \\
0 & \cdots & \cdots & \cdots & 0 & \frac{k}{m_{n}} & \frac{k}{m_{n}}
\end{array}\right] \text {, } \\
& A_{22}=\left[\begin{array}{ccccccc}
\frac{-b}{m_{1}} & \frac{b}{m_{1}} & 0 & \cdots & \cdots & \cdots & 0 \\
\frac{b}{m_{2}} & \frac{-2 b}{m_{2}} & \frac{b}{m_{2}} & 0 & \cdots & \cdots & 0 \\
0 & \frac{b}{m_{3}} & \frac{-2 b}{m_{3}} & \frac{b}{m_{3}} & 0 & \cdots & 0 \\
0 & \cdots & \cdots & \cdots & \cdots & \cdots & 0 \\
0 & \cdots & \cdots & \cdots & 0 & \frac{b}{m_{n}} & \frac{b}{m_{n}}
\end{array}\right] \text {, } \\
& B_{2}=\operatorname{diag}\left(\begin{array}{llll}
\frac{\lambda_{1}}{m_{1}} & \frac{\lambda_{2}}{m_{2}} & \cdots & \frac{\lambda_{n}}{m_{n}}
\end{array}\right) \text {, }
\end{aligned}
$$

$E_{02}$

$$
\begin{aligned}
& =\operatorname{diag}\left(\frac{g \sin \theta_{1}+0.002 d_{a}}{R_{t}} \cdots \cdots \frac{g \sin \theta_{n}+0.002 d_{a}}{R_{t}}\right), \\
& C_{02}=\left[\begin{array}{lllll}
-c_{0} & -c_{0} & \cdots & \cdots & -c_{0}
\end{array}\right]^{\mathrm{T}}, \\
& C_{v 2}=\operatorname{diag}\left(\begin{array}{lllll}
-c_{v} & -c_{v} & \cdots & \cdots & -c_{v}
\end{array}\right) \text {, }
\end{aligned}
$$

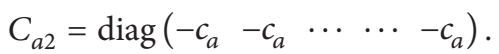

2.3. Fault Analysis of Traction Systems in HST. In HST, velocity cruise control is accomplished through the traction system which consists of high-voltage circuit (included by pantograph, current transformer, main circuit breaker, etc.),

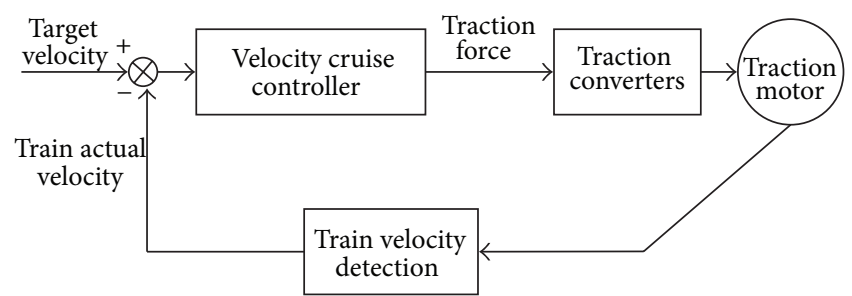

FIGURE 2: The principle of velocity cruise control in HST.

traction transformer, traction converter, asynchronous motor, wheels, and so on [12-14]. The principle of velocity cruise control is shown in Figure 2.

Traction converter is used to control traction motor to achieve velocity cruise control of HST. The probability of incorrect voltage and current, such as malfunction of electronic components in traction converter, is great $[56,57]$. The output of $i$ th actuator in failure status can be described as $u_{f}(t)$ and its elements is shown as follows:

$$
u_{i}^{f}(t)=f_{i}(t) u_{i}(t) \quad(i=1, \ldots, n),
$$

where $u_{i}(t)$ is control input without AF. $f_{i}(t) \in\left(f_{i}, \bar{f}_{i}\right)$ is unknown piecewise hopping function, with known boundary $\underline{f}_{i}, \bar{f}_{i}$, which represents malfunction of electronic components in traction system and

$$
f_{i}(t)= \begin{cases}1 & t<t_{1} \\ f_{i 1} & t_{1} \leq t<t_{2} \\ f_{i 2} & t_{2} \leq t<t_{3} \\ \vdots & \vdots\end{cases}
$$

where $f_{i j}$ is unknown constant for $j=1,2, \ldots, l$ and $t_{j}$ for $j=1,2, \ldots, l$ satisfies

$$
t_{j}-t_{j-1} \geq t_{j, \min }
$$

where $t_{0}=0$ and $t_{j, \min }$, which is the minimum switch time for $f_{i j}$, will be discussed later. It is assumed that $E_{02}$ is unknown constant matrix for $t \in\left(t_{j-1}, t_{j}\right)$. After AF, dynamic model (10) will be

$$
\dot{x}(t)=A x(t)+B f(t) u(t)+C_{0}+C_{v} x(t)+C_{a} x^{2}(t),
$$

or suppose there are $p$ failed actuators; we have

$$
\begin{aligned}
\dot{x}(t)= & A x(t)+\sum_{i=1, \ldots, m} b_{i} f_{i}(t) u_{i}(t)+C_{0}+C_{v} x(t)+C_{a} x^{2}(t) \\
= & A x(t)+\sum_{i \neq j_{1}, \ldots, j_{p}} b_{i} u_{i}(t)+\sum_{j=j_{1}, \ldots, j_{p}} f_{j}(t) b_{j} u_{j}(t)+C_{0} \\
& +C_{v} x(t)+C_{a} x^{2}(t)
\end{aligned}
$$

where $b_{i}$ is the column of $B, f(t) \in R^{n \times n}$, and

$$
f(t)=\operatorname{diag}\left(f_{1}(t) \quad f_{2}(t) \quad f_{3}(t) \cdots f_{m}(t)\right) .
$$


Therefore, after AF, HST system is multiplied by an unknown AF which impacts on stability of HST system. The control objective is to design a feedback control such that all signals in the closed-loop system are bounded, and $x(t)$ asymptotically tracks a given reference $x_{m}(t)$ which is generated from the reference system:

$$
\dot{x}_{m}=A_{m} x_{m}(t)+B_{m} r(t)
$$

where $x_{m} \in R^{2 n}, A_{m} \in R^{2 n \times 2 n}$, and $B_{m} \in R^{2 n \times 1}$ are known constant matrices such that all the eigenvalues of $A_{m}$ are in the open left-half complex plane, and $r(t) \in R$ is bounded and piecewise continuous.

\section{I\&I Adaptive Fault-Tolerant Control Based on AMFDD}

In this section, an adaptive controller combined with faulttolerant module based on I\&I AMFDD technology is developed. I\&I AMFDD module is designed to detect AF and direct adaptive controller handles AF of HST. The system (10) can be rewritten as follows:

$$
\dot{x}(t)=A x(t)+B u(t)+\theta \Phi(x),
$$

where $\theta=\left[\begin{array}{lll}C_{0} & C_{v} & C_{a}\end{array}\right]$, and $\Phi(x)=\left[\begin{array}{lll}1 & x(t) & x^{2}(t)\end{array}\right]^{\mathrm{T}}$ is Lipschitz with $G$ as

$$
\begin{aligned}
& \left|\Phi\left(x_{1}\right)-\Phi\left(x_{2}\right)\right| \\
& =\left|\left[\begin{array}{lll}
1 & x_{1}(t) & x_{1}^{2}(t)
\end{array}\right]^{\mathrm{T}}-\left[\begin{array}{lll}
1 & x_{2}(t) & x_{2}^{2}(t)
\end{array}\right]^{\mathrm{T}}\right|
\end{aligned}
$$

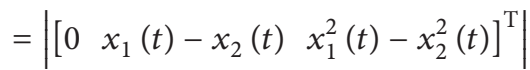

$$
\begin{aligned}
& =\left|x_{1}(t)-x_{2}(t)\right|\left|\left[\begin{array}{lll}
0 & 1 & x_{1}(t)+x_{2}(t)
\end{array}\right]^{\mathrm{T}}\right| \\
& \leq\left|\left[\begin{array}{lll}
0 & 1 & 2 \bar{x}
\end{array}\right]^{\mathrm{T}}\right|\left|x_{1}(t)-x_{2}(t)\right| \\
& =G\left|x_{1}(t)-x_{2}(t)\right| \text {, }
\end{aligned}
$$

where $\bar{x}$ is the maximum value of position and velocity of HST. For the control problem considered in this paper, the following is assumed.

Assumption 2. The state of system (19) is available at every instant, and the maximum value of position and velocity of HST, that is, $\bar{x}$, is known before AF (12).

Assumption 3. If system parameters and AF (up to $n-1$ failures) are known, the remaining actuators can still achieve a desired control objective.

Assumption 4. All pairs $\{A, B f(t)\}$ are uniformly completely controllable for $f_{i}(t) \in\left(\underline{f}_{i}, \bar{f}_{i}\right), i=1, \ldots, n$.

3.1. Adaptive Model-Based Fault Detection and Diagnosis (AMFDD). AMFDD module is combined with train control system in HST. Taking China Railways High-speed (CRH) trains [58-60] as an example, AMFDD module is designed to detect AF in Train Control and Management System. For $t \in\left(t_{j-1}, t_{j}\right)$, consider the following AMFDD:

$$
\dot{\hat{x}}(t)=A \widehat{x}(t)+\widehat{\Theta} \Phi(\widehat{x}(t))+B u(t)-L(y(t)-\widehat{y}(t)),
$$

where $\widehat{x}, \widehat{\Theta}$, and $\widehat{y}$ are the estimate of $x, \theta$, and $y$. I\&I adaptive control is a noncertainty equivalence technique with

$$
\widehat{\Theta}=\widehat{\theta}+\beta(x),
$$

where the estimation objective is to render the manifold

$$
\{(x, \widehat{\theta}), \mid \widehat{\theta}+\beta(x)-\theta=0\}
$$

invariant and (asymptotically) attractive, and the continuous function $\beta(x)$, which is added to the estimated parameter vector $\hat{\theta}$, is also a design parameter. Estimation of $\theta$ is achieved by driving the estimate error

$$
z=\widehat{\theta}-\theta+\beta(x)
$$

to zero. Define parameterized function:

$$
Q_{p}(\theta)=\frac{d \beta(x)}{d x} \theta \Phi(x),
$$

which satisfies $P(x)$ - monotone, that is,

$$
(a-b) P(x)\left(Q_{p}(a)-Q_{p}(b)\right) \geq 0,
$$

where $P(x)$ is a design function, which simplifies computational complexity of $\beta(x)$, satisfying

$$
P(x) \geq 0, \quad \frac{d P(x)}{d t} \leq 0 .
$$

Theorem 5. AMFDD (21) and the adaptive law

$$
\dot{\hat{\theta}}=-\frac{d \beta(x)}{d x}(A x(t)+(\widehat{\theta}+\beta(x(t))) \Phi(x(t))+B u(t))
$$

can realise that

(1) the estimate error (24) converges to zero, that is, $\lim _{t \rightarrow \infty} z=0 ;$

(2) the observational error $e_{x}$ satisfies

$$
e_{x}(t) \leq e_{\max }(t)
$$

where

$$
\begin{aligned}
e_{x}(t)= & x(t)-\widehat{x}(t), \\
e_{\max }(t)= & e^{\lambda_{\max }(\bar{A}) t} e_{0} \\
& +e^{-\lambda_{\min }(\bar{A}) t} \bar{\theta} \Phi(\bar{x}) \bar{A}^{-1}\left(e^{\lambda_{\max }(\bar{A}) t}-1\right),
\end{aligned}
$$

where $e_{0}$ is initial state vector of $e_{x}, \bar{\theta}$ is the upper bound of $z$, and $\lambda_{\max }()$ and $\lambda_{\min }()$ denote the maximum and minimum value of matrix. 
(3) Decision on the occurrence of AF in carriages is made if at least one term of the estimation error $e_{x}(t)$ exceeds its corresponding error bound $e_{\max }(t)$.

Proof. The derivative of $z$ is as follows:

$$
\dot{z}=\dot{\hat{\theta}}+\frac{d \beta(x)}{d x}(A x(t)+\theta \Phi(x(t))+B u(t)),
$$

which clearly yields

$$
\begin{aligned}
\dot{z} & =\dot{\hat{\theta}}+\frac{d \beta(x)}{d x}(A x(t)+\theta \Phi(x(t))+B u(t)) \\
& =-\frac{d \beta(x)}{d x}(\hat{\theta}+\beta(x(t))) \Phi(x(t))+\frac{d \beta(x)}{d x} \theta \Phi(x(t)) \\
& =Q_{p}(\theta)-Q_{p}(\widehat{\theta}+\beta(x(t))) .
\end{aligned}
$$

Consider a positive-definite function $V=(1 / 2) z^{T} P(x) z$, and using (26), (27), and (32), we have

$$
\begin{aligned}
\dot{V}= & z^{T} P(x) \dot{z}+\frac{1}{2} z^{T} \dot{P}(x) z \\
= & z^{T} P(x)\left(\dot{\hat{\theta}}+\frac{d \beta(x)}{d x}(A x(t)+\theta \Phi(x(t))\right. \\
& \quad+B u(t)))+\frac{1}{2} z^{T} \frac{d P(x)}{d t} z \\
\leq & (\widehat{\theta}-\theta+\beta(x))^{T} P(x) \quad \\
& \times\left(Q_{p}(\theta)-Q_{p}(\widehat{\theta}+\beta(x))\right) \leq 0
\end{aligned}
$$

from which convergence of $z(t)$ follows; that is,

$$
\lim _{t \rightarrow \infty}(\widehat{\theta}+\beta(x))=\theta .
$$

The derivative of $e_{x}$ is as follows:

$$
\begin{aligned}
\dot{e}_{x}(t)= & \dot{x}(t)-\dot{\hat{x}}(t) \\
= & A x(t)+\theta \Phi(x)+B u(t) \\
& -A \widehat{x}(t)-(\widehat{\theta}+\beta(\widehat{x})) \Phi(\widehat{x}) \\
& -B u(t)-L(y(t)-\widehat{y}(t)) \\
= & (A-L C) e_{x}(t)+\theta \Phi(x)-(\widehat{\theta}+\beta(\widehat{x})) \Phi(\widehat{x}) \\
= & (A-L C) e_{x}(t)+\theta \Phi(x)-(\widehat{\theta}+\beta(\widehat{x})) \Phi(x) \\
& +(\widehat{\theta}+\beta(\widehat{x})) \Phi(x)-(\widehat{\theta}+\beta(\widehat{x})) \Phi(\widehat{x}) \\
\leq & (A-L C) e_{x}(t)-z \Phi(x)+(\widehat{\theta}+\beta(\widehat{x})) G e_{x}(t) \\
= & (A-L C+(\widehat{\theta}+\beta(\widehat{x})) G) e_{x}(t)-z \Phi(x) \\
= & \bar{A} e_{x}(t)-z \Phi(x) .
\end{aligned}
$$

Consequently, when no AF occurs, we have

$$
\begin{aligned}
e_{x}(t)= & e_{x}^{\bar{A} t} e_{0}+\int_{0}^{t} e_{x}^{-\bar{A}(t-\tau)}(-z(\tau) \Phi(x(\tau))) d \tau \\
\leq & e_{x}^{\lambda_{\max }(\bar{A}) t} e_{0}+e_{x}^{-\lambda_{\min }(\bar{A}) t} \int_{0}^{t} e_{x}^{\bar{A} \tau}(-z(\tau) \Phi(x)) d \tau \\
\leq & e_{x}^{\lambda_{\max }(\bar{A}) t} e_{0}+e_{x}^{-\lambda_{\min }(\bar{A}) t} \bar{\theta} \Phi(\bar{x}) \bar{A}^{-1} \int_{0}^{t} e_{x}^{\bar{A} \tau} d \bar{A} \tau \\
= & e_{x}^{\lambda_{\max }(\bar{A}) t} e_{0}+e_{x}^{-\lambda_{\min }(\bar{A}) t} \bar{\theta} \Phi(\bar{x}) \bar{A}^{-1} \\
& \times\left(e_{x}^{\lambda_{\max }(\bar{A}) t}-1\right)=e_{\max } .
\end{aligned}
$$

Hence, decision on the occurrence of AF in carriages is made if at least one term of the estimation error $e_{x}(t)$ exceeds its corresponding error bound $e_{\max }(t)$.

3.2. Adaptive Control for Unknown AF. After detection of AF, the key task of $\mathrm{AF}$ compensation control is to design a direct adaptive control law such that all trajectories of the closedloop system (16) are bounded and $\lim _{t \rightarrow \infty} x(t)=x_{m}$. Using the AMFDD (21), the AF system (16) is as follows:

$$
\begin{aligned}
\dot{x}(t)= & A x(t)+\sum_{i=1}^{n} b_{i} f_{i}(t) u_{i}(t)+z \Phi(x) \\
= & A x(t)+\sum_{i \neq j_{1}, \ldots, j_{p}} b_{i} u_{i}(t) \\
& +\sum_{j=j_{1}, \ldots, j_{p}} f_{j}(t) b_{j} u_{j}(t)+z \Phi(x) \\
= & A x(t)+B^{\prime}(t) u(t)+z \Phi(x),
\end{aligned}
$$

where $B^{\prime}(t)=\left[\begin{array}{lllll}b_{1}^{\prime} & b_{2}^{\prime} & b_{3}^{\prime} & \cdots & b_{n}^{\prime}\end{array}\right] \in R^{2 n \times n}$ is unknown constant matrix with $f(t), t \in\left(t_{j-1}, t_{j}\right)$, and $z$, which is exponential convergence, is defined in (24).

Before addressing the control problem for unknown AF, we must first derive the existence of controllers for the system (37) with known AF to obtain some basic plant-model matching conditions which are useful for controller parameterization in adaptive designs for unknown AF. Without AF, matching equations of HST system (37) are satisfied:

$$
\begin{gathered}
A+\sum_{i=1}^{n} b_{i} K_{1 i}^{* T}=A_{m}, \\
\sum_{i=1}^{n} b_{i} k_{2 i}^{*}=B_{m},
\end{gathered}
$$

and $K_{1}^{*}=\left[\begin{array}{llll}K_{11}^{*} & K_{12}^{*} & \cdots & K_{1 n}^{*}\end{array}\right] \in R^{2 n \times n}$ and $k_{2}^{*}=$ $\left[\begin{array}{llll}k_{21}^{*} & k_{22}^{*} & \cdots & k_{2 n}^{*}\end{array}\right]^{T} \in R^{n}$. We see Assumptions 3 implies that there exist constant vectors $K_{s 1 j}^{*}$ and nonzero constant 
$k_{s 2 j}^{*}$ such that when only one AF occurs, the following matching equations are satisfied:

$$
\begin{gathered}
A+\sum_{i \neq j}^{n} b_{i} K_{1 i}^{* T}+b_{j}^{\prime} K_{s 1 j}^{* T}=A_{m}, \\
\sum_{i \neq j}^{n} b_{i} k_{2 i}^{*}+b_{j}^{\prime} k_{s 2 j}^{*}=B_{m} .
\end{gathered}
$$

Using (38) and (39), we obtain

$$
\begin{gathered}
b_{j}^{\prime}=-\frac{k_{2 j}^{*}}{k_{s 2 j}^{*}} b_{j}=-\frac{K_{1 j}^{* T}}{K_{s 1 j}^{* T}} b_{j}, \\
\frac{K_{1 j}^{* T}}{k_{2 j}^{*}}=\frac{K_{s 1 j}^{* T}}{k_{s 2 j}^{*}} .
\end{gathered}
$$

Now we develop an adaptive control scheme for the system (37) with unknown AF. We propose the controller structure:

$$
u(t)=K_{1}^{T}(t) x(t)+k_{2}(t) r(t)+k_{3}(t),
$$

where

$$
\begin{gathered}
K_{1}(t)=\left[\begin{array}{llll}
K_{11}(t) & K_{12}(t) & \cdots & K_{1 n}(t)
\end{array}\right] \in R^{2 n \times n}, \\
k_{2}(t)=\left[\begin{array}{lllll}
k_{21}(t) & k_{22}(t) & \cdots & k_{2 n}(t)
\end{array}\right]^{T} \in R^{n}
\end{gathered}
$$

are adaptive estimates of the unknown parameters $K_{1}^{*}$ and $k_{2}^{*}$, and

$$
k_{3}(t)=\left[\begin{array}{lllll}
k_{31}(t) & k_{32}(t) & \cdots & k_{3 n}(t)
\end{array}\right]^{T} \in R^{n}
$$

is a design vector. Define the parameter errors

$$
\begin{aligned}
& e(t)=x(t)-x_{m}(t), \\
& \widetilde{K}_{1 i}(t)=K_{1 i}(t)-K_{1 i}^{*}, \\
& \widetilde{k}_{2 i}(t)=k_{2 i}(t)-k_{2 i}^{*},
\end{aligned}
$$

for $i=1, \ldots, n$. Substituting the control law (41) into the system (37), we obtain

$$
\begin{aligned}
\dot{x}(t)= & A x(t)+B^{\prime}(t) u(t)+z \Phi(x) \\
= & A x(t)+B^{\prime} K_{1}^{T}(t) x(t)+B^{\prime} k_{2}(t) r(t) \\
& +z \Phi(x)+B^{\prime} k_{3}(t) .
\end{aligned}
$$

Using (40) and (44), the system (45) is as follows:

$$
\begin{aligned}
\dot{x}(t)= & \left(A+B^{\prime} K_{1}^{T}\right) x(t)+B^{\prime} k_{2} r(t)+z \Phi(x)+B^{\prime} k_{3} \\
= & \left(A+B^{\prime} K_{1}^{* T}\right) x(t)+B^{\prime} k_{2}^{*} r(t)+B^{\prime} \widetilde{K}_{1}^{T}(t) x(t) \\
& +B^{\prime} \widetilde{k}_{2}(t) r(t)+z \Phi(x)+B^{\prime} k_{3}(t) \\
= & A_{m} x(t)+B_{m} r(t)+B^{\prime} \widetilde{K}_{1}^{T}(t) x(t) \\
& +B^{\prime} \widetilde{k}_{2}(t) r(t)+z \Phi(x)+B^{\prime} k_{3}(t) \\
= & A_{m} x(t)+B_{m} r(t)+\sum_{i=1}^{n} \frac{-k_{2 i}^{*}}{k_{s 2 i}^{*}} b_{i} \widetilde{K}_{1 i}^{T}(t) x(t) \\
& +\sum_{i=1}^{n} \frac{-k_{2 i}^{*}}{k_{s 2 i}^{*}} b_{i} \widetilde{k}_{2 i}(t) r(t)+z \Phi(x) \\
& +\sum_{i=1}^{n} \frac{-k_{2 i}^{*}}{k_{s 2 i}^{*}} b_{i} k_{3 i}(t) .
\end{aligned}
$$

Then, we have the tracking error equation:

$$
\begin{aligned}
\dot{e}(t)= & \dot{x}(t)-\dot{x}_{m}(t) \\
= & A_{m} e(t)+\sum_{i=1}^{n} \frac{-k_{2 i}^{*}}{k_{s 2 i}^{*}} b_{i} \widetilde{K}_{1 i}^{T}(t) x(t) \\
& +\sum_{i=1}^{n} \frac{-k_{2 i}^{*}}{k_{s 2 i}^{*}} b_{i} \widetilde{k}_{2 i}(t) r(t) \\
& +z \Phi(x)+\sum_{i=1}^{n} \frac{-k_{2 i}^{*}}{k_{s 2 i}^{*}} b_{i} k_{3 i}(t) .
\end{aligned}
$$

Consider a positive definite function

$$
\begin{aligned}
V= & e^{T}(t) P e(t)+\sum_{i=1}^{n} \frac{1}{\left|k_{s 2 i}^{*}\right|} \widetilde{K}_{1 i}^{T}(t) \Gamma_{1 i}^{-1} \widetilde{K}_{1 i}(t) \\
& +\sum_{i=1}^{n} \frac{1}{\left|k_{s 2 i}^{*}\right|} \widetilde{k}_{2 i}^{2}(t) \gamma_{2 i}^{-1} .
\end{aligned}
$$

According to [25], we assume that the sign of parameter $k_{s 2 j}^{*}$ is known, and $k_{s 2 j}^{0}$ is a known upper bound on $\left|k_{s 2 j}^{*}\right|$. Choose the adaptive laws as

$$
\begin{gathered}
\dot{K}_{1 i}(t)=-\operatorname{sgn}\left[k_{s 2 i}^{*}\right] \Gamma_{1 i} x e^{T}(t) P B_{m}, \\
\dot{k}_{2 i}(t)=-\operatorname{sgn}\left[k_{s 2 i}^{*}\right] \gamma_{2 i} r(t) e^{T}(t) P B_{m},
\end{gathered}
$$

where $P \in R^{2 n \times 2 n}, P=P^{T}>0$ such that

$$
P A_{m}+A_{m}^{T} P=-Q
$$


for any constant $Q \in R^{2 n \times 2 n}$ such that $Q=Q^{T}>0, \Gamma_{1 i}$ is constant matrix such that $\Gamma_{1 i}=\Gamma_{1 i}^{T}>0$, and $\gamma_{2 i}>0$ is constant. Then, we have

$$
\begin{aligned}
\dot{V} \leq & -e^{T}(t) Q e(t)+2 e^{T}(t) P \bar{\theta} \Phi(x) \\
& +2 e^{T}(t) P \sum_{i=1}^{n} \frac{-k_{2 i}^{*}}{k_{s 2 i}^{*}} b_{i} k_{3 i}(t)
\end{aligned}
$$

where $\bar{\theta}$ is defined in (29). We choose

$$
k_{3 i}(t)=\operatorname{sgn}\left[k_{s 2 i}^{*}\right] k_{i}^{0}|\Phi(x)|^{2} e^{T}(t) P B_{m},
$$

and we obtain

$$
\begin{aligned}
\dot{V} \leq & -e^{T}(t) Q e(t)+2 e^{T}(t) P \bar{\theta} \Phi(x) \\
& -2 e^{T}(t) P B \sum_{i=1}^{n} \frac{k_{2 i}^{*}}{k_{s 2 i}^{*}} k_{3 i}(t) \\
= & -e^{T}(t) Q e(t)+\frac{|\bar{\theta}|^{2}\|P\|^{2}}{2\left|e^{T}(t) P B_{m}\right|^{2} \sum_{i=1}^{n}\left(1 /\left|k_{s 2 i}^{*}\right|\right) k_{i}^{0}}|e(t)|^{2} \\
& -\frac{|\bar{\theta}|^{2}\|P\|^{2}}{2\left|e^{T}(t) P B_{m}\right|^{2} \sum_{i=1}^{n}\left(1 /\left|k_{s 2 i}^{*}\right|\right) k_{i}^{0}}|e(t)|^{2} \\
& +2\left|e^{T}(t) P \bar{\theta} \Phi(x)\right|-2\left|e^{T}(t) P B_{m}\right|^{2} \sum_{i=1}^{n} \frac{1}{\left|k_{s 2 i}^{*}\right|} k_{i}^{0}|\Phi(x)|^{2} \\
= & -e^{T}(t) Q e(t)+\frac{|\bar{\theta}|^{2}\|P\|^{2}}{2\left|e^{T}(t) P B_{m}\right|^{2} \sum_{i=1}^{n}\left(1 /\left|k_{s 2 i}^{*}\right|\right) k_{i}^{0}}|e(t)|^{2} \\
& -\left(\frac{|\bar{\theta}|\|P\|}{\sqrt{2\left|e^{T}(t) P B_{m}\right|^{2} \sum_{i=1}^{n}\left(1 /\left|k_{s 2 i}^{*}\right|\right) k_{i}^{0}}}|e(t)|\right. \\
\leq & -e^{T}(t) Q e(t)+\phi_{0}, \quad \sqrt{2 \mid e^{2}\|P\|^{2}} \\
& \left.-\sqrt{2\left|e^{T}(t) P B_{m}\right|^{2} \sum_{i=1}^{n} \frac{1}{\left|k_{s 2 i}^{*}\right|_{i}} k_{i}^{0}|\Phi(x)|}\right)^{2} \\
& \left.(t) B_{m}\right|^{2} \sum_{i=1}^{n}\left(1 /\left|k_{s 2 i}^{*}\right|\right) k_{i}^{0}
\end{aligned}
$$

where

$$
\phi_{0}=\frac{|\bar{\theta}|^{2}}{2\left|B_{m}\right|^{2} \sum_{i=1}^{n}\left(1 /\left|k_{s 2 i}^{0}\right|\right) k_{i}^{0}} .
$$

Hence, $\dot{V} \leq 0$ whenever $|e|>\sqrt{\phi_{0} / \lambda_{\min }(Q)}$, which means that the tracking error $e(t)$ will converge to a set of a small size if $k_{i}^{0}$ is chosen to be large.
Theorem 6. For HST system (37) with Assumptions 1-4 and AF (12) satisfying (56), the controller is given by the control law (41) and the adaptation laws (49) and (52); then we have the following.

(1) HST system (37) is internally stable and all closed-loop signals are bounded.

(2) If $k_{i}^{0}$ is chosen to be large, the tracking error $e(t)$ will converge to a set of a small size.

Remark 7. The convergence time $t_{e}$ is defined by

$$
t_{e}=\left\{t_{e}|| e(t) \mid \leq \delta\right\},
$$

where $\delta$ is a design parameter, and we assume the minimum switch time $t_{\min }$ must satisfy

$$
t_{j, \min }>t_{e} .
$$

3.3. Adaptive Control for Unknown $A F$ and CIS. In this subsection, we consider unknown AF (12) and CIS simultaneously for HST system (37). In many practical applications, CIS occurs after AF (12), for example, when signal $u(t)$ generated by the adaptive fault-tolerant control law (41) cannot be implemented due to some physical constraints in HST traction system.

Due to CIS, adaptive fault-tolerant control law, which is different from (41), is as follows:

$$
u^{s}(t)=\operatorname{sat}\left(u(t), u_{\min }, u_{\max }\right),
$$

where $u_{\min }$ and $u_{\max }$ are the minimum and maximum values of $u(t)$, and $u(t)$ is defined in (41). Saturation function $\operatorname{sat}\left(u(t), u_{\min }, u_{\max }\right)$ is linear with unity slope between its lower and upper bound; that is,

$$
\operatorname{sat}\left(u(t), u_{\min }, u_{\max }\right)= \begin{cases}u_{\min }, & u(t)<u_{\min }, \\ u(t), & u_{\min } \leq u(t) \leq u_{\max } \\ u_{\max }, & u(t)>u_{\max },\end{cases}
$$

and we consider CIS error $\eta=\left[\begin{array}{llll}\eta_{1} & \eta_{2} & \cdots & \eta_{n}\end{array}\right]^{T} \in R^{n}$ described by

$$
\eta_{i}(t)=u_{i}^{s}(t)-u_{i}(t)
$$

and $\left|\eta_{i}(t)\right|<\bar{\eta}$. Now we develop an adaptive control scheme for the system (37) with unknown AF (12) and CIS (57). We suppose there are $q$ CIS in HST, that is, $j=j_{1}, \ldots, j_{q}$, and propose the controller structure:

$$
u_{j}^{s}(t)=\left\{\begin{array}{r}
K_{1 j}^{T}(t) x(t)+k_{2 j}(t) r(t)+k_{4 j}(t)+k_{3 j}(t) \\
j \neq j_{1}, \ldots, j_{q} \\
K_{1 j}^{T}(t) x(t)+k_{2 j}(t) r(t)+\eta_{j}(t)+k_{3 j}(t) \\
j=j_{1}, \ldots, j_{q},
\end{array}\right.
$$


where $K_{1 j}(t), k_{2 j}(t)$, and $k_{3 j}(t)$ are defined in (49) and (52). $k_{4}=\left[\begin{array}{llll}k_{41} & k_{42} & \cdots & k_{4 n}\end{array}\right]^{T} \in R^{n}$. We obtain

$$
\begin{aligned}
\dot{x}(t)= & A x(t)+B^{\prime}(t) u^{s}(t)+z \Phi(x) \\
= & A_{m} x(t)+B_{m} r(t)+\sum_{i=1}^{n} \frac{-k_{2 i}^{*}}{k_{s 2 i}^{*}} b_{i} \widetilde{K}_{1 i}^{T}(t) x(t) \\
& +\sum_{i=1}^{n} \frac{-k_{2 i}^{*}}{k_{s 2 i}^{*}} \widetilde{k}_{i}(t) r(t)+\sum_{i=1}^{n} \frac{-k_{2 i}^{*}}{k_{s 2 i}^{*}} b_{i} k_{3 i}(t)+z \Phi(x) \\
& +\sum_{j \neq j_{1}, \ldots, j_{q}} \frac{-k_{2 j}^{*}}{k_{s 2 j}^{*}} b_{j} k_{4 j}(t)+\sum_{j=j_{1}, \ldots, j_{q}} \frac{-k_{2 j}^{*}}{k_{s 2 j}^{*}} b_{j} \eta_{j}(t) .
\end{aligned}
$$

We have the tracking error equation:

$$
\begin{aligned}
\dot{e}(t)= & \dot{x}(t)-\dot{x}_{m}(t) \\
= & A_{m} e(t)+\sum_{i=1}^{n} \frac{-k_{2 i}^{*}}{k_{s 2 i}^{*}} b_{i} \widetilde{K}_{1 i}^{T}(t) x(t) \\
& +\sum_{i=1}^{n} \frac{-k_{2 i}^{*}}{k_{s 2 i}^{*}} b_{i} \widetilde{k}_{2 i}(t) r(t)+\sum_{i=1}^{n} \frac{-k_{2 i}^{*}}{k_{s 2 i}^{*}} b_{i} k_{3 i}(t) \\
& +z \Phi(x)+\sum_{j \neq j_{1}, \ldots, j_{q}} \frac{-k_{2 j}^{*}}{k_{s 2 j}^{*}} b_{j} k_{4 j}(t) \\
& +\sum_{j=j_{1}, \ldots, j_{q}} \frac{-k_{2 j}^{*}}{k_{s 2 j}^{*}} b_{j} \eta_{j}(t) .
\end{aligned}
$$

Consider a positive definite function:

$$
\begin{aligned}
V= & e^{T}(t) \operatorname{Pe}(t)+\sum_{i=1}^{n} \frac{1}{\left|k_{s 2 i}^{*}\right|} \widetilde{K}_{1 i}^{T}(t) \Gamma_{1 i}^{-1} \widetilde{K}_{1 i}(t) \\
& +\sum_{i=1}^{n} \frac{1}{\left|k_{s 2 i}^{*}\right|} \widetilde{k}_{2 i}^{2}(t) \gamma_{2 i}^{-1},
\end{aligned}
$$

and using (49) and (52), we have

$$
\begin{array}{r}
\dot{V} \leq-e^{T}(t) Q e(t)+\frac{|\bar{\theta}|^{2}\|P\|^{2}}{2\left|e^{T}(t) P B_{m}\right|^{2} \sum_{i=1}^{n}\left(1 /\left|k_{s 2 i}^{0}\right|\right) k_{i}^{0}}|e(t)|^{2} \\
+2 e^{T}(t) P B\left(\sum_{j \neq j_{1}, \ldots, j_{q}} \frac{-k_{2 j}^{*}}{k_{s 2 j}^{*}} k_{4 j}(t)+\sum_{j=j_{1}, \ldots, j_{q}} \frac{-k_{2 j}^{*}}{k_{s 2 j}^{*}} \eta_{j}(t)\right) \\
\leq-e^{T}(t) Q e(t)+\phi_{0}+2 e^{T}(t) P B\left(\sum_{j \neq j_{1}, \ldots, j_{q}} \frac{-k_{2 j}^{*}}{k_{s 2 j}^{*}} k_{4 j}(t)\right. \\
\left.+\sum_{j=j_{1}, \ldots, j_{q}} \frac{-k_{2 j}^{*}}{k_{s 2 j}^{*}} \eta_{j}(t)\right),
\end{array}
$$

and we assume

$$
\begin{gathered}
\eta_{p}=\max _{j_{1}, \ldots, j_{q} \in\{1, \ldots, n\}}\left|\sum_{j=j_{1}, \ldots, j_{q}} \frac{-k_{2 j}^{*}}{k_{s 2 j}^{*}} \eta_{j}(t)\right|(1 \leq q \leq n-1), \\
\eta^{0}=\max \left\{\eta_{1}, \ldots, \eta_{n-1}\right\}, \\
k_{s 2 j}^{0}>\left|k_{s 2 j}^{*}\right| .
\end{gathered}
$$

We choose the design signal $k_{4 j}(t)$ as

$$
k_{4 j}(t)=k_{s 2 j}^{0} \eta_{0} \operatorname{sgn}\left[e^{T}(t) P B_{m}\right] \operatorname{sgn}\left[k_{s 2 j}^{*}\right] .
$$

Since the control signal $k_{4 j}(t)$ in (66) is not continuous, according to $[61,62]$, to avoid system chatterings caused by such discontinuous control laws, the following common approximations will be used for $\operatorname{sgn}(\cdot)$ function:

$$
\operatorname{sgn}[x] \approx \frac{x}{|x|+\varepsilon}, \quad \varepsilon>0 .
$$

We obtain

$$
\begin{aligned}
\dot{V} \leq & -e^{T}(t) Q e(t)+\phi_{0}-2 \frac{\left|e^{T}(t) P B_{m}\right|^{2}}{\left|e^{T}(t) P B_{m}\right|+\varepsilon} \\
& \times \sum_{j \neq j_{1}, \ldots, j_{q} \mid} \frac{k_{s 2 j}^{0}}{\left|k_{s 2 j}^{*}\right|} \eta_{0}+2\left|e^{T}(t) P B_{m}\right| \eta_{p} \\
\leq & -e^{T}(t) Q e(t)+\phi_{0}-2\left|e^{T}(t) P B_{m}\right| \\
& \times\left(\frac{\left|e^{T}(t) P B_{m}\right|}{\left|e^{T}(t) P B_{m}\right|+\varepsilon} \sum_{j \neq j_{1}, \ldots, j_{q} \mid} \frac{k_{s 2 j}^{0}\left|k_{s 2 j}^{*}\right|}{\left.\eta_{0}-\eta_{p}\right)}\right. \\
\leq & -e^{T}(t) Q e(t)+\phi_{0}+2 \eta_{0}\left|e^{T}(t) P B_{m}\right| \\
& \times \frac{\varepsilon}{\left|e^{T}(t) P B_{m}\right|+\varepsilon} \sum_{j \neq j_{1}, \ldots, j_{q}} \frac{k_{s 2 j}^{0}}{\left|k_{s 2 j}^{*}\right|} \\
\leq & -e^{T}(t) Q e(t)+\phi_{0}+2 \beta_{0} \varepsilon,
\end{aligned}
$$

where $\beta_{0}=\sum_{j \neq j_{1}, \ldots, j_{q}}\left(k_{s 2 j}^{0} /\left|k_{s 2 j}^{*}\right|\right) \eta_{0}$ is a constant. Hence $\dot{V}<$ 0 , whenever

$$
|e|>\sqrt{\frac{\phi_{0}+2 \beta_{0} \varepsilon}{\lambda_{\min }(Q)}},
$$

which means $|e|$ decreases to a lower bound proportional to $\sqrt{\varepsilon}$ and $k_{i}^{0}$.

Theorem 8. For HST system (37) with Assumptions 1-4 and $A F(12)$ and CIS (57), the controller is given by the control law (60) and the adaptation laws (49), (52), and (66); then we have the following.

(1) HST system (37) is internally stable and all closed-loop signals are bounded.

(2) If $\sqrt{\varepsilon}$ is chosen to be small and $k_{i}^{0}$ is chosen to be large, the tracking error $e(t)$ will converge to a set of a small size. 


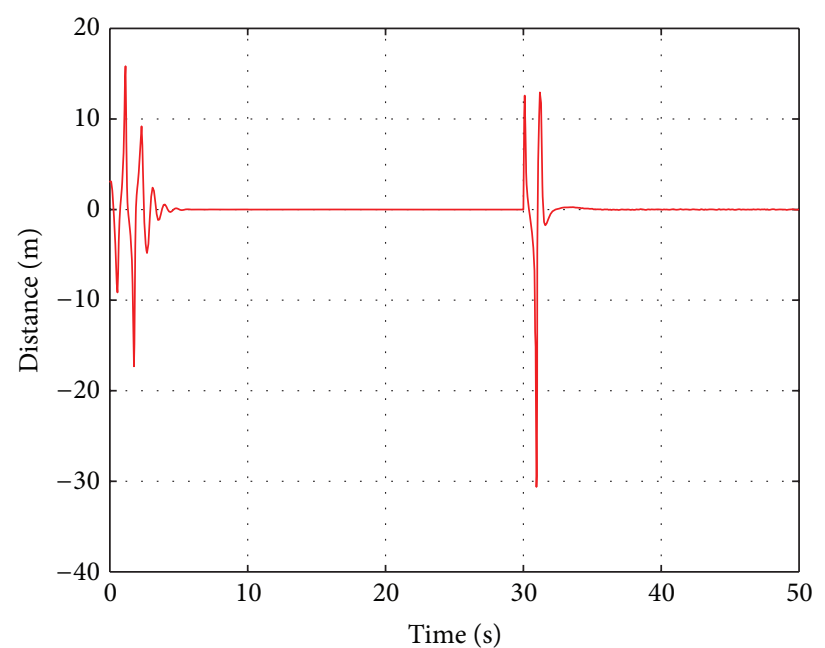

(a)

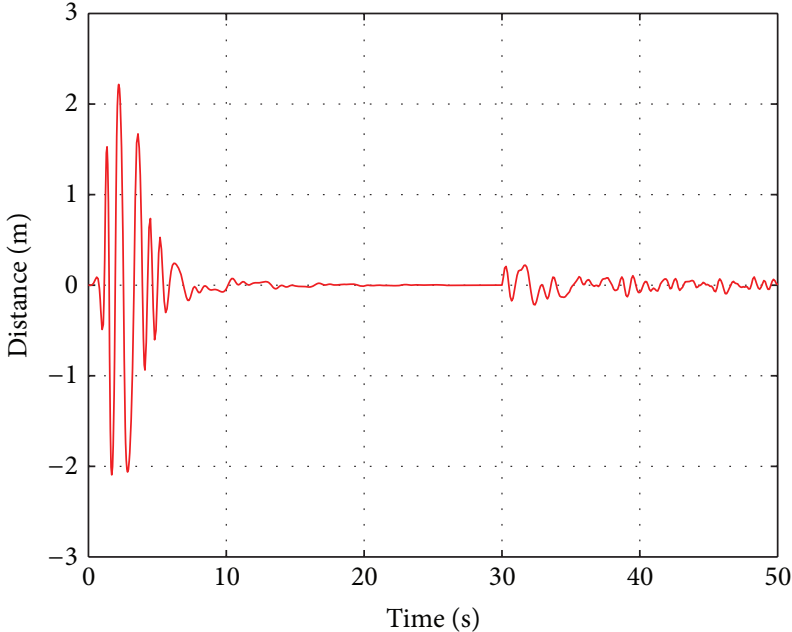

(b)

FIGURE 3: The position tracking error of the carriages.

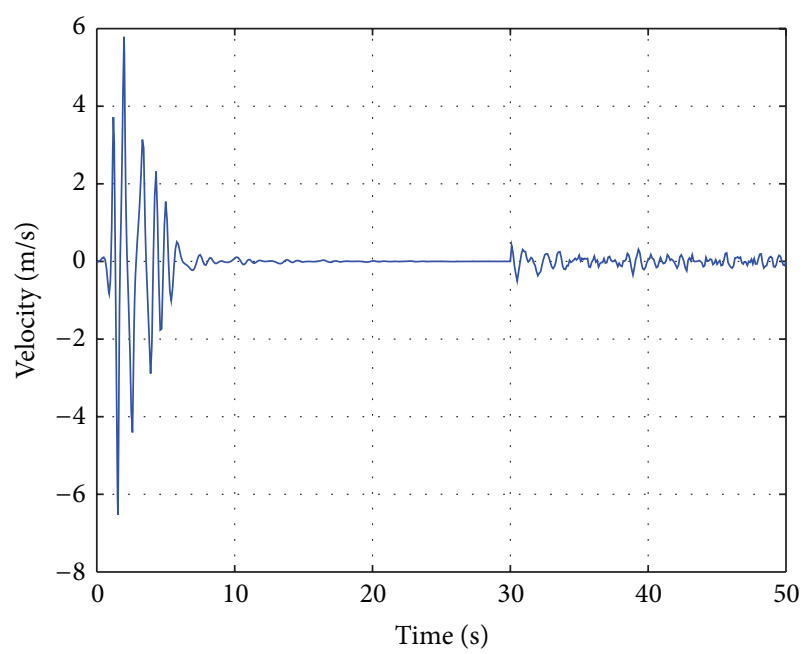

(a)

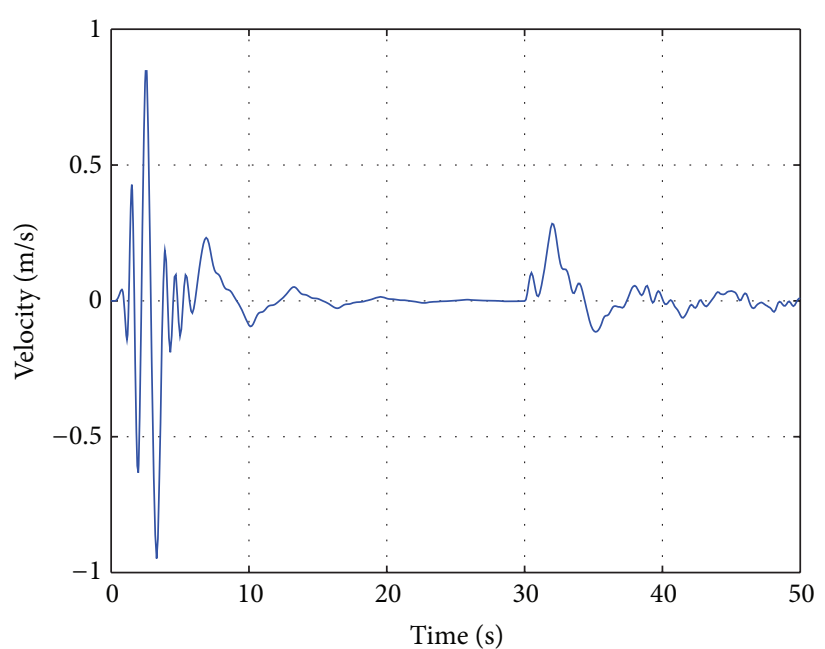

(b)

FIGURE 4: The velocity tracking error of the carriages.

\section{Simulations}

To prove the effectiveness of proposed controller, a train model of CRH2 [59], which consists of two powered carriages, is used. The parameters in this simulation are given in Table 1.

The unknown parameters, which should not be ignored, are shown as follows:

$$
\begin{gathered}
c_{0}=5.2 \mathrm{~N} / \mathrm{kg}, \\
c_{v}=0.038 \mathrm{~N} \cdot \mathrm{s} /(\mathrm{m} \cdot \mathrm{kg}), \\
c_{a}=0.00112 \mathrm{~N}^{2} \cdot \mathrm{s} /\left(\mathrm{m}^{2} \cdot \mathrm{kg}\right) .
\end{gathered}
$$

We consider the AF in the first carriage is as follows:

$$
f_{1}(t) u_{1}(t)= \begin{cases}1, & 0 \leq t<30 \\ 0.6, & 30 \leq t \leq 50\end{cases}
$$

and CIS in the second carriage is as follows:

$$
u_{2}(t) \in(-40 k N, 40 k N) \text {. }
$$

In order to exhibit the advantage of the proposed adaptive fault-tolerant control, a fault-tolerant control using neural networks [63] (FTCNN) is introduced. Using FTCNN, performance of the system (37) under AF (71) and CIS (72) is shown in Figures 3 and 4 . The maximum position and velocity tracking errors after $\mathrm{AF}$ attain to $2 \mathrm{~m}$ and $2.5 \mathrm{~m} / \mathrm{s}$, respectively.

The AMFDD (21) and adaptive fault-tolerant control law (60) with adaptation laws (49), (52), and (66) are applied to 
TABLE 1: Parameters of high-speed trains.

\begin{tabular}{lcc}
\hline Symbol & Implication & Value \\
\hline$n$ & Number of carriages & 2 \\
$m_{i}(i=1,2)$ & Mass of powered carriages & $8500 \mathrm{~kg}$ \\
$k$ & Elasticity coefficient & $2 \times 10^{7} \mathrm{~N} / \mathrm{m}$ \\
$b$ & Damping coefficient & $5 \times 10^{6} \mathrm{~N} \cdot \mathrm{s} / \mathrm{m}$ \\
\hline
\end{tabular}

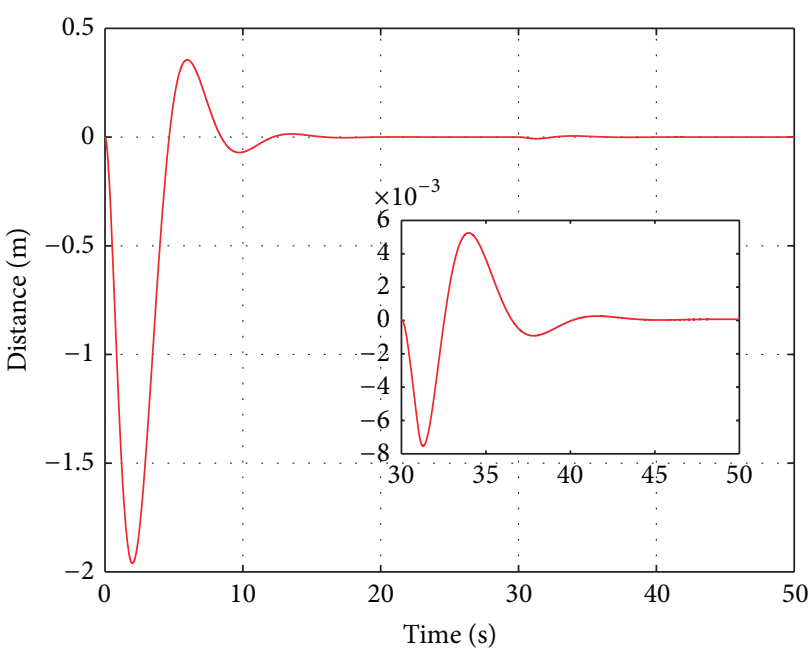

(a)



(b)

FIgURE 5: The position tracking error of the carriages.

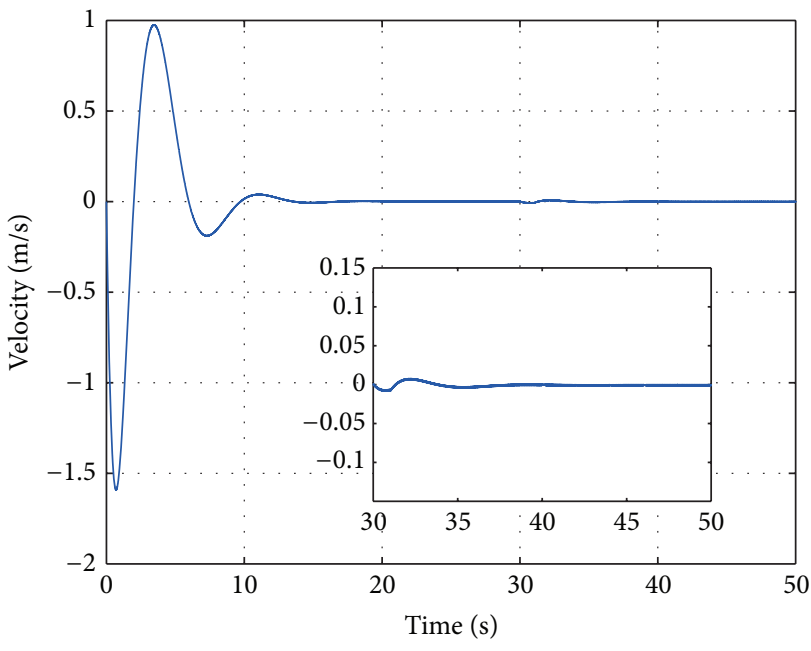

(a)

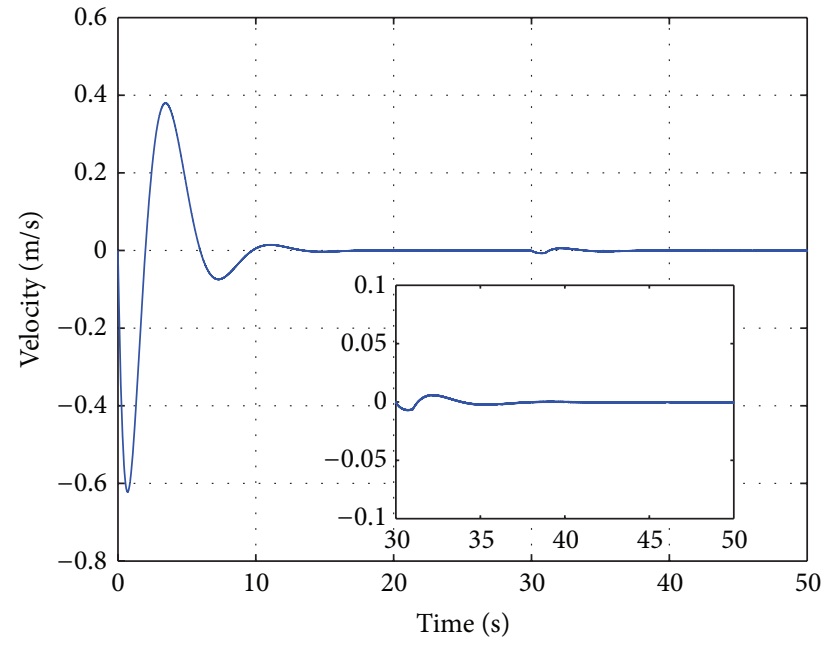

(b)

FIGURE 6: The velocity tracking error of the carriages.

handle unknown AF (71) and CIS (72) in HST system. Figures 5 and 6 show the system responses which indicate that even when there are unknown AF, the tracking errors still converge to zero. It is shown that the developed adaptive fault-tolerant control law (60) with adaptation laws (49), (52), and (66) ensures that, in addition to closed-loop signal boundedness, the position and velocity tracking errors converge to zero as the time goes by, despite of the plant uncertainties, AF, and CIS.

\section{Conclusion}

In this paper, an adaptive state feedback and tracking control with AMFDD module is proposed to deal with a class of 
HST in the presence of unknown CIS and AF. That is, some of the plant inputs are influenced by hopping function. An AMFDD module using I\&I observer is introduced based on the model of HST to detect AF, and a corresponding adaptive control law based on the AMFDD information is switched on to realize the fault-tolerant control. The proposed adaptive FTC guarantees the boundedness of all signals in the HST system.

\section{Conflict of Interests}

The authors declare that there is no conflict of interests regarding the publication of this paper.

\section{References}

[1] T. Zhang and M. Guay, "Adaptive control for a class of secondorder nonlinear systems with unknown input nonlinearities," IEEE Transactions on Systems, Man, and Cybernetics B: Cybernetics, vol. 33, no. 1, pp. 143-149, 2003.

[2] S. S. Ge and C. Wang, "Adaptive neural control of uncertain MIMO nonlinear systems," IEEE Transactions on Neural Networks, vol. 15, no. 3, pp. 674-692, 2004.

[3] S. Labiod, M. S. Boucherit, and T. M. Guerra, "Adaptive fuzzy control of a class of MIMO nonlinear systems," Fuzzy Sets and Systems, vol. 151, no. 1, pp. 59-77, 2005.

[4] Q. Hu, G. Ma, and L. Xie, "Robust and adaptive variable structure output feedback control of uncertain systems with input nonlinearity," Automatica, vol. 44, no. 2, pp. 552-559, 2008.

[5] M. Chen, S. S. Ge, and B. V. E. How, "Robust adaptive neural network control for a class of uncertain MIMO nonlinear systems with input nonlinearities," IEEE Transactions on Neural Networks, vol. 21, no. 5, pp. 796-812, 2010.

[6] M. Chen, S. S. Ge, and B. Ren, "Adaptive tracking control of uncertain MIMO nonlinear systems with input constraints," Automatica, vol. 47, no. 3, pp. 452-465, 2011.

[7] S. Tong and Y. Li, "Adaptive fuzzy output feedback tracking backstepping control of strict-feedback nonlinear systems with unknown dead zones," IEEE Transactions on Fuzzy Systems, vol. 20, no. 1, pp. 168-180, 2012.

[8] H. Zhang, Y. Shi, and M. Liu, " $H_{\infty}$ step tracking control for networked discrete-time nonlinear systems with integral and predictive actions," IEEE Transactions on Industrial Informatics, vol. 9, no. 1, pp. 337-345, 2013.

[9] Q. Zhou, P. Shi, S. Xu, and H. Li, "Adaptive output feedback control for nonlinear time-delay systems by fuzzy approximation approach," IEEE Transactions on Fuzzy Systems, vol. 21, no. 2, pp. 301-313, 2013.

[10] Y. Liu, S. Tong, and C. L. P. Chen, "Adaptive fuzzy control via observer design for uncertain nonlinear systems with unmodeled dynamics," IEEE Transactions on Fuzzy Systems, vol. 21, no. 2, pp. 275-288, 2013.

[11] H. Li, J. Yu, C. Hilton, and H. Liu, "Adaptive sliding-mode control for nonlinear active suspension vehicle systems using TS fuzzy approach," IEEE Transactions on Industrial Electronics, vol. 60, no. 8, pp. 3328-3338, 2013.

[12] X. Deng, "Traction drive system of $200 \mathrm{~km} / \mathrm{h}$ CRH 2 EMUs," Electric Drive for Locomotives, vol. 4, no. 1, pp. 31-38, 2008.

[13] H. Wang, Y. Wang, C. Xie, and X. Hu, "Reliability modeling and assigning for CRH2 electric multiple unit," Journal of the China Railway Society, vol. 5, no. 1, pp. 19-21, 2009.
[14] G. Xie and Q. Lu, "Modeling and simulation at temporary power-off state of EMU traction driver system," Jidian Gongcheng Mechanical \& Electrical Engineering Magazine, vol. 28, no. 5, pp. 605-609, 2011.

[15] X. Zhuan and X. Xia, "Fault-tolerant control of heavy-haul trains,” Vehicle System Dynamics, vol. 48, no. 6, pp. 705-735, 2010.

[16] X. Zhuan and X. Xia, "Speed regulation with measured output feedback in the control of heavy haul trains," Automatica, vol. 44, no. 1, pp. 242-247, 2008.

[17] X. Liu and Y. Song, "Robust adaptive fault-tolerant control of dynamic systems with floating nonlinearities and fading actuators," in Proceedings of the Chinese Control and Decision Conference (CCDC '11), pp. 149-154, Mianyang, China, May 2011.

[18] Q. Song and Y. Song, "Data-based fault-tolerant control of highspeed trains with traction/braking notch nonlinearities and actuator failures," IEEE Transactions on Neural Networks, vol. 22, no. 12, pp. 2250-2261, 2011.

[19] H. Dong, S. Gao, B. Ning, and L. Li, "Extended fuzzy logic controller for high speed train," Neural Computing and Applications, vol. 22, no. 2, pp. 321-328, 2013.

[20] H. Yang, Y. Fu, K. Zhang, and Z. Li, "Speed tracking control using an ANFIS model for high-speed electric multiple unit," Control Engineering Practice, vol. 23, pp. 57-65, 2014.

[21] M. Faieghi, A. Jalali, and S. K. M. Mashhadi, "Robust adaptive cruise control of high speed trains," ISA Transactions, vol. 53, no. 2, pp. 533-541, 2014.

[22] H. Yang, V. Cocquempot, and B. Jiang, "Robust fault tolerant tracking control with application to hybrid nonlinear systems," IET Control Theory and Applications, vol. 3, no. 2, pp. 211-224, 2009.

[23] B. Jiang, Z. Gao, P. Shi, and Y. Xu, "Adaptive fault-tolerant tracking control of near-space vehicle using TakagiSugeno fuzzy models," IEEE Transactions on Fuzzy Systems, vol. 18, no. 5, pp. 1000-1007, 2010.

[24] Y. Xu, B. Jiang, G. Tao, and Z. Gao, "Fault tolerant control for a class of nonlinear systems with application to near space vehicle," Circuits, Systems, and Signal Processing, vol. 30, no. 3, pp. 655-672, 2011.

[25] G. Tao, S. M. Joshi, and X. Ma, "Adaptive state feedback and tracking control of systems with actuator failures," IEEE Transactions on Automatic Control, vol. 46, no. 1, pp. 78-95, 2001.

[26] G. Tao, S. Chen, and S. M. Joshi, "An adaptive actuator failure compensation controller using output feedback," IEEE Transactions on Automatic Control, vol. 47, no. 3, pp. 506-511, 2002.

[27] X. Tang, G. Tao, and S. M. Joshi, "Adaptive actuator failure compensation for parametric strict feedback systems and an aircraft application," Automatica, vol. 39, no. 11, pp. 1975-1982, 2003.

[28] Z. Qu, C. M. Ihlefeld, Y. Jin, and A. Saengdeejing, "Robust faulttolerant self-recovering control of nonlinear uncertain systems," Automatica, vol. 39, no. 10, pp. 1763-1771, 2003.

[29] Z. Wang, G. Wei, and G. Feng, "Reliable $H_{\infty}$ control for discrete-time piecewise linear systems with infinite distributed delays," Automatica, vol. 45, no. 12, pp. 2991-2994, 2009.

[30] M. J. Khosrowjerdi, "Mixed $H_{2} / H_{\infty}$ approach to fault-tolerant controller design for Lipschitz non-linear systems," IET Control Theory and Applications, vol. 5, no. 2, pp. 299-307, 2011. 
[31] Y. Shen, L. Liu, and E. H. Dowell, "Adaptive fault-tolerant robust control for a linear system with adaptive fault identification," IET Control Theory \& Applications, vol. 7, no. 2, pp. 246-252, 2013.

[32] Q. Shen, B. Jiang, and V. Cocquempot, "Fuzzy logic systembased adaptive fault-tolerant control for near-space vehicle attitude dynamics with actuator faults," IEEE Transactions on Fuzzy Systems, vol. 21, no. 2, pp. 289-300, 2013.

[33] M. T. Hamayun, C. Edwards, and H. Alwi, "A fault tolerant control allocation scheme with output integral sliding modes," Automatica, vol. 49, no. 6, pp. 1830-1837, 2013.

[34] Q. Shen, B. Jiang, and V. Cocquempot, "Adaptive fault-tolerant backstepping control against actuator gain faults and its applications to an aircraft longitudinal motion dynamics," International Journal of Robust and Nonlinear Control, vol. 23, no. 15, pp. 17531779, 2013.

[35] M. S. Mahmoud, A. M. Memon, and P. Shi, "Observer-based fault-tolerant control for a class of nonlinear networked control systems," International Journal of Control, vol. 87, no. 8, pp. 17071715, 2014.

[36] S. Gao, H. Dong, Y. Chen, B. Ning, and G. Chen, "Adaptive and robust automatic train control systems with input saturation," Control and Intelligent Systems, vol. 41, no. 2, pp. 103-111, 2013.

[37] S. Gao, H. Dong, Y. Chen, B. Ning, G. Chen, and X. Yang, "Approximation-based robust adaptive automatic train control: an approach for actuator saturation," IEEE Transactions on Intelligent Transportation Systems, vol. 14, no. 4, pp. 1733-1742, 2013.

[38] S. P. Karason and A. M. Annaswamy, "Adaptive control in the presence of input constraints," IEEE Transactions on Automatic Control, vol. 39, no. 11, pp. 2325-2330, 1994.

[39] A. R. Teel, "A nonlinear small gain theorem for the analysis of control systems with saturation," IEEE Transactions on Automatic Control, vol. 41, no. 9, pp. 1256-1270, 1996.

[40] M. Polycarpou, J. Farrell, and M. Sharma, "On-line approximation control of uncertain nonlinear systems: issues with control input saturation," in Proceedings of the American Control Conference, pp. 543-548, Denver, Colo, USA, June 2003.

[41] Y. Chang, Y. Wang, M. Hung, and P. Chen, "Regional stability and $\mathrm{H} \infty$ performance control of an input-saturated induction motor via LMI approach," Asian Journal of Control, vol. 7, no. 4, pp. 368-379, 2005.

[42] F. Wu, Z. Lin, and Q. Zheng, "Output feedback stabilization of linear systems with actuator saturation," IEEE Transactions on Automatic Control, vol. 52, no. 1, pp. 122-128, 2007.

[43] B. Zhou, G. Duan, and Z. Lin, "A parametric Lyapunov equation approach to the design of low gain feedback," IEEE Transactions on Automatic Control, vol. 53, no. 6, pp. 1548-1554, 2008.

[44] B. Zhou, Z. Lin, and G. Duan, "A parametric Lyapunov equation approach to low gain feedback design for discrete-time systems," Automatica, vol. 45, no. 1, pp. 238-244, 2009.

[45] E. Fridman and M. Dambrine, "Control under quantization, saturation and delay: an LMI approach," Automatica, vol. 45, no. 10, pp. 2258-2264, 2009.

[46] J. Huang, C. Wen, W. Wang, and Z. Jiang, "Adaptive stabilization and tracking control of a nonholonomic mobile robot with input saturation and disturbance," Systems \& Control Letters, vol. 62 , no. 3, pp. 234-241, 2013.

[47] A. Astolfi and R. Ortega, "Immersion and invariance: a new tool for stabilization and adaptive control of nonlinear systems," IEEE Transactions on Automatic Control, vol. 48, no. 4, pp. 590606, 2003.
[48] X. Liu, R. Ortega, H. Su, and J. Chu, "Immersion and invariance adaptive control of nonlinearly parameterized nonlinear systems," IEEE Transactions on Automatic Control, vol. 55, no. 9, pp. 2209-2214, 2010.

[49] X. Liu, R. Ortega, H. Su, and J. Chu, "On adaptive control of nonlinearly parameterized nonlinear systems: towards a constructive procedure," Systems and Control Letters, vol. 60, no. 1, pp. 36-43, 2011.

[50] C. Yang and Y. Sun, "Mixed $\mathrm{H}_{2} / \mathrm{H}_{\infty}$ cruise controller design for high speed train," International Journal of Control, vol. 74, no. 9, pp. 905-920, 2001.

[51] C. Yang and Y. Sun, "Robust cruise control of high speed train with hardening/softening nonlinear coupler," in Proceedings of the American Control Conference (ACC '99), pp. 2200-2204, San Diego, Calif, USA, June 1999.

[52] H. K. Khalil and J. W. Grizzle, Nonlinear Systems, Prentice Hall, Upper Saddle River, NJ, USA, 3rd edition, 2002.

[53] G. F. Franklin and J. Powell, Feedback Control of Dynamic Systems, Addison Wesley, New York, NY, USA, 1994.

[54] R. S. Raghunathan, H. D. Kim, and T. Setoguchi, "Aerodynamics of high-speed railway train," Progress in Aerospace Sciences, vol. 38, no. 6-7, pp. 469-514, 2002.

[55] V. K. Garg and R. V. Dukkipati, Dynamics of Railway Vehicle Systems, Academic Press, Toronto, Canada, 1984.

[56] J.-M. Thébaud, E. Woirgard, C. Zardini, S. Azzopardi, O. Briat, and J. Vinassa, "Strategy for designing accelerated aging tests to evaluate IGBT power modules lifetime in real operation mode," IEEE Transactions on Components and Packaging Technologies, vol. 26, no. 2, pp. 429-438, 2003.

[57] A. Ginart, M. J. Roemer, P. W. Kalgren, and K. Goebel, "Modeling aging effects of IGBTs in power drives by ringing characterization," in Proceedings of the International Conference on Prognostics and Health Management (PHM '08), pp. 1-7, Denver, Colo, USA, October 2008.

[58] S. Zhang, CRH1-EMU, China Railway, Beijing, China, 2008.

[59] S. Zhang, CRH2-EMU, China Railway Publishing House, Beijing, China, 2008.

[60] S. Zhang, CRH5 -EMU, China Railway Publishing House, Beijing, China, 2008.

[61] A. F. Filippov, "Differential equations with discontinuous right hand side," Matematicheskii Sbornik, vol. 51, no. 1, pp. 199-231, 1960.

[62] V. I. Utkin, Sliding Modes in Control Optimization, Springer, Berlin, Germany, 1991.

[63] M. R. Napolitano, Y. An, and B. A. Seanor, "A fault tolerant flight control system for sensor and actuator failures using neural networks," Aircraft Design, vol. 3, no. 2, pp. 103-128, 2000. 




Advances in

Operations Research

mansans

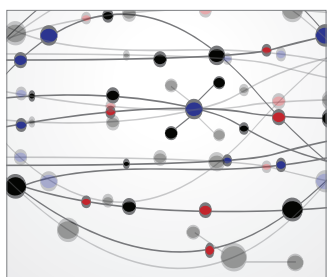

The Scientific World Journal
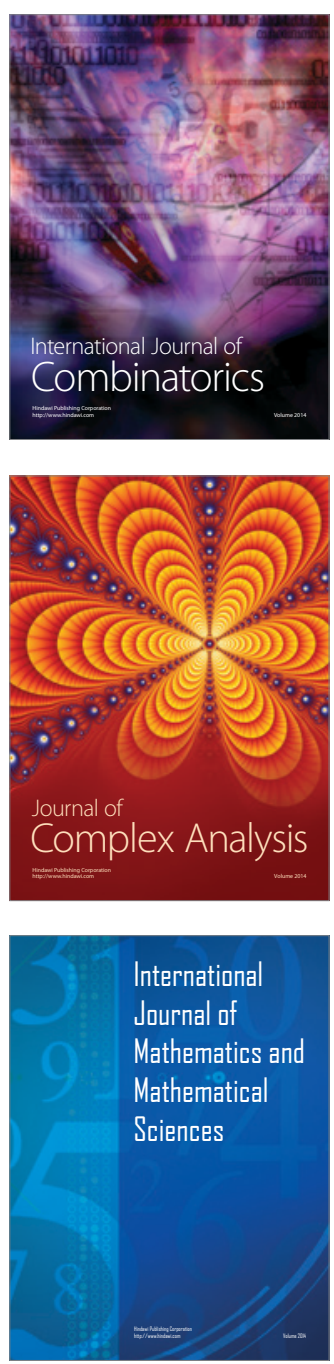
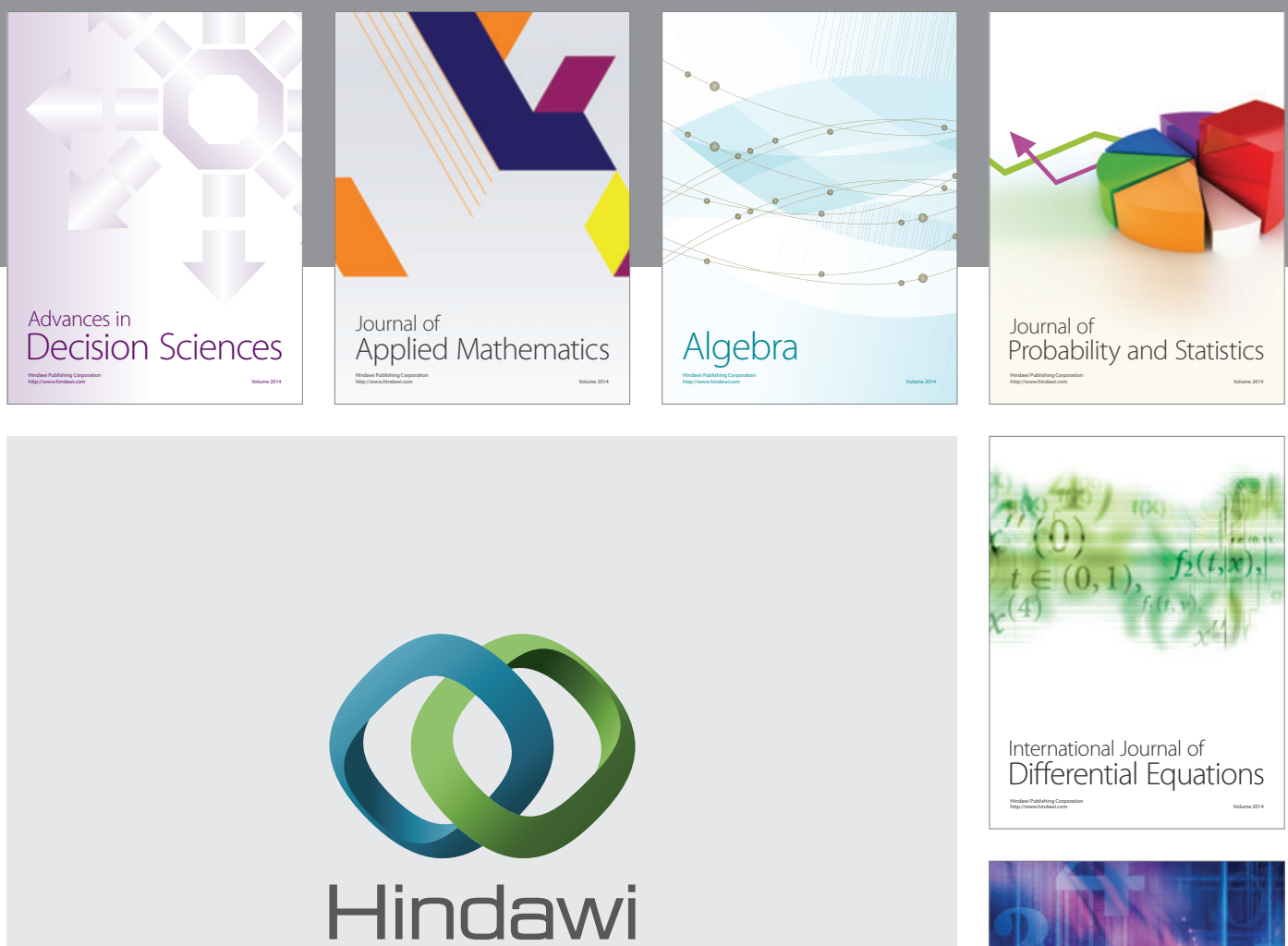

Submit your manuscripts at http://www.hindawi.com


Journal of

Function Spaces




